\title{
The Effect of a Hand-Stretching Device During the Management of Spasticity in Chronic Hemiparetic Stroke Patients
}

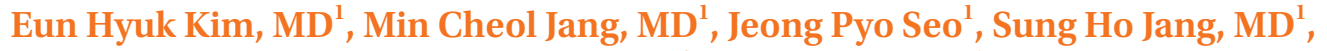 \\ Jun Chan Song, $\mathrm{PhD}^{2}$, Hae Min Jo, $\mathrm{MD}^{1}$
}

\begin{abstract}
${ }^{1}$ Department of Physical Medicine and Rehabilitation, Yeungnam University College of Medicine, Daegu;
${ }^{2}$ Department of Physical Therapy, Daegu Health College, Daegu, Korea
\end{abstract}

Objective To describe a hand-stretching device that was developed for the management of hand spasticity in chronic hemiparetic stroke patients, and the effects of this device on hand spasticity.

Methods Fifteen chronic hemiparetic stroke patients with finger flexor spasticity were recruited and randomly assigned to an intervention group (8 patients) or a control group (7 patients). The stretching device consists of a resting hand splint, a finger and thumb stretcher, and a frame. In use, the stretched state was maintained for 10 minutes per exercise session, and the exercise was performed twice daily for 4 weeks. Spasticity of finger flexor muscles in the two groups was assessed 3 times, 4 weeks apart, using the Modified Ashworth Scale (MAS). Patients in the intervention group were assessed twice (pre-1 and pre-2) before and once (post-1) after starting the stretching program.

Results Mean MAS (mMAS) scores at initial evaluations were not significantly different at pre- 1 in the intervention group and at lst assessment in the control group ( $p>0.05$ ). In addition, no significant differences were observed between mMAS scores at pre- 1 and pre- 2 in the intervention group ( $>0.05)$. However, mMAS scores at post1 were significantly lower than that at pre- 2 in the intervention group $(\mathrm{p}<0.05)$. Within the control group, no significant changes in mMAS scores were observed between 1st, 2nd, and 3rd assessments ( $p>0.05)$. In addition, mMAS scores at post- 1 in the intervention group were significantly decreased compared with those at the 3rd assessment in the control group $(\mathrm{p}<0.05)$.

Conclusion The devised stretching device was found to relieve hand spasticity effectively in chronic hemiparetic stroke patients.

Keywords Muscle spasticity, Hand, Stretch, Stroke, Hemiparesis

Received April 29, 2012; Accepted July 11, 2012

Corresponding author: Hae Min Jo

Department of Physical Medicine and Rehabilitation, Yeungnam University College of Medicine, 170 Hyeonchung-ro, Nam-gu, Daegu 705-717, Korea

Tel: +82-53-620-3456, Fax: +82-53-625-3508, E-mail: jhm090@hanmail. net

(c) This is an open-access article distributed under the terms of the Creative Commons Attribution Non-Commercial License (http://creativecommons. org/licenses/by-nc/3.0) which permits unrestricted noncommercial use, distribution, and reproduction in any medium, provided the original work is properly cited.

Copyright $\odot 2013$ by Korean Academy of Rehabilitation Medicine

\section{INTRODUCTION}

Spasticity is defined as a velocity-dependent increase in tonic stretch reflex with exaggerated tendon reflex following upper motor neuron injury [1]. Approximately $65 \%$ of all stroke patients experience spasticity [2], which can result in functional disability due to muscle tightness and joint stiffness of the affected extremity [3-5]. Because of its high prevalence and close relationship with functional 
outcome, appropriate management of spasticity is an important issue during the rehabilitation of stroke patients.

Several modalities including medication, stretching exercise, nerve block, muscle wash, neuromuscular electrical stimulation, repetitive transcranial magnetic stimulation, serial cast, antispastic brace, and surgery have been used to manage spasticity in stroke [6-12]. Of these, stretching exercises that involve movements of joints through their ranges of motion (ROM) by an external force are basic management techniques [13,14]. This type of exercise can be performed manually, and is frequently used because it is easy performed, has fewer side effects, and is cheap $[15,16]$. However, manual stretching exercises require a therapist to perform repetitive exercises on a regular basis, and outcomes depend on therapist experience $[13,17]$. To overcome these drawbacks, some stretching devices have been developed, and their effectiveness has been demonstrated $[13,14,17,18]$.

We recently developed a stretching device for the management of hand spasticity and our research team, Jung et al. [18] demonstrated the beneficial effects of this device in 21 chronic stroke patients. However, this device was found to have some limitations in chronic stroke patients. First, it was difficult to maintain the hand firmly in the stretched state because the string fixator tends to be loose. In addition, chronic hemiparetic stroke patients are required to pull the strings of the device to stretch hands, and they found it difficult to handle the device alone. Finally, frequent changes between the stretched and relaxed states every 30 seconds for 20 minutes led to poor compliance. To resolve these issues, we improved the stretching device and simplified the protocol. In the present study, we evaluated the effect of the modified device and protocol on hand spasticity in chronic hemiparetic stroke patients.

\section{MATERIALS AND METHODS}

\section{Subjects}

Among the patients admitted for treatment at our university hospital department of rehabilitation, 15 consecutive hemiparetic stroke patients ( 10 males, 5 females; mean, $51.2 \pm 11.4$ years; range, 29 to 72 years) were recruited according to the following criteria: 1) $\geq 6$ months after stroke onset; 2) complete weakness (zero: no joint motion, no palpable muscle contraction) or severe weakness of the affected finger flexor (trace: palpable muscle contraction, but no visible movement); 3) finger flexor spasticity (a Modified Ashworth Scale [MAS] score [19, 20 ] of $>1$ ); 4) an age of 18 to 75 years; 5) no history of peripheral nerve injury or musculoskeletal disease (e.g., arthritis, musculotendinous injury or bone fracture) in
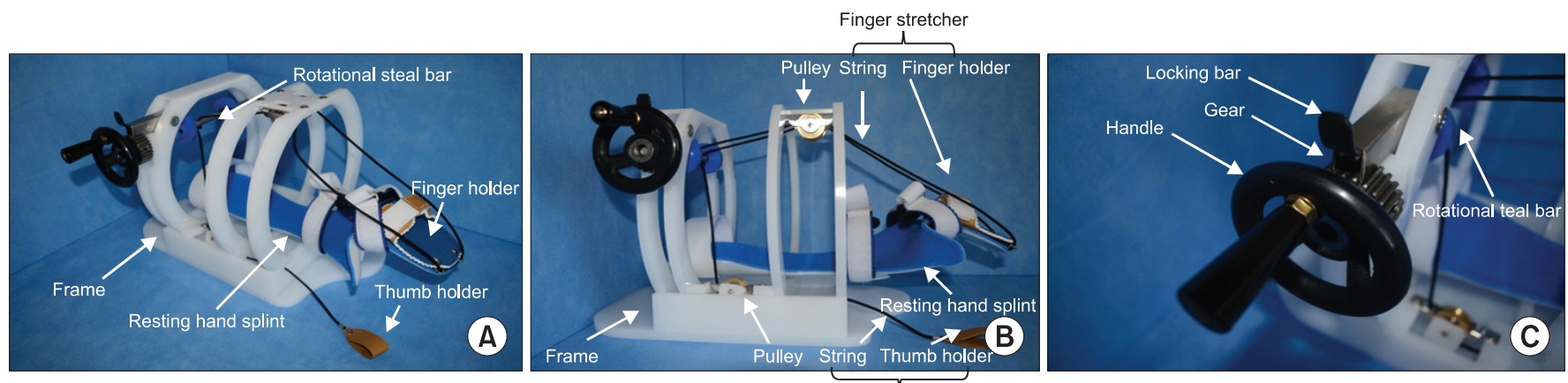

Thumb stretcher
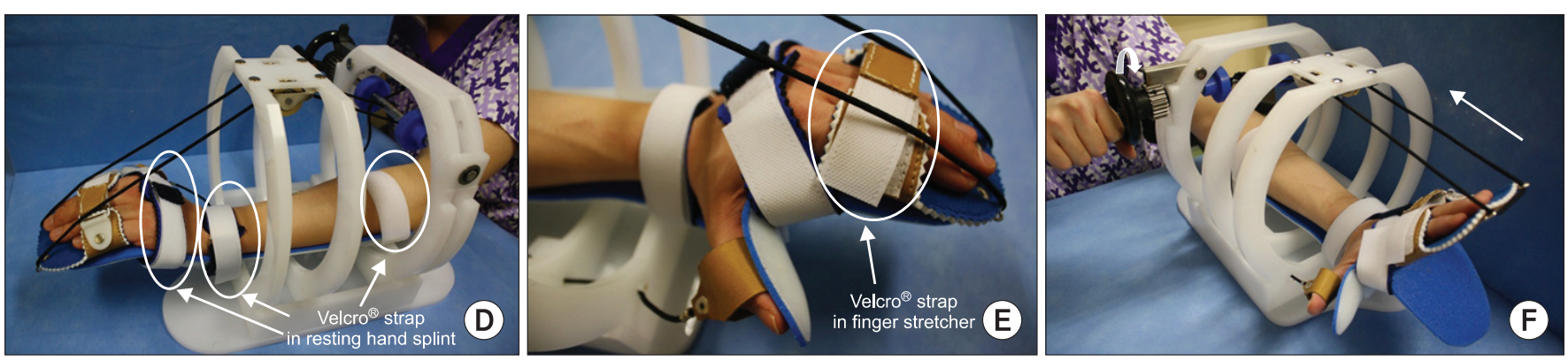

Fig. 1. Stretching device. 
the affected upper extremity; 6) no contracture of the affected wrist or fingers; and 7) no history of any invasive procedure (Botox, alcohol, or phenol) for the treatment of spasticity for at least 6 months before the start of this study. Patients with apraxia, somatosensory problems, or cognitive problems (Mini-Mental State Examination score of $<25$ ) were excluded. Patients were randomly assigned either to an intervention group (8 patients; mean age, $47.7 \pm 8.0$ years) or to a control group ( 7 patients; mean age, $55.1 \pm 14.0$ years). We did not change any drugs or perform any procedures that might have affected spasticity during the study period. All patients provided written informed consent and the study protocol was approved by our Institutional Review Board.

\section{Stretching device}

Fundamentally, the modified stretching device consists of a resting hand splint, a finger and thumb stretcher, and a frame similar to that of the previous stretching device [18]. To achieve firm hand fixation during stretching and to improve handling of the device, a rotational steel bar connected to a gear equipped with a ratchet is included. The resting hand splint is fixed to the bed of the frame (Fig. 1A). The finger-stretching unit consists of a finger holder (a rectangular box) for the 4 fingers (from the 2nd to 5th digits), and 2 strings. The finger holder is connected by strings to two pulleys fixed beneath the crossbars of the frame. The thumb-stretching unit, which consists of a leather thumb holder and a string, is located on the medial side of the side-bar and is connected to a pulley fixed above the bed of the frame by a string (Fig. 1B). A rotational steel bar attached to the gear assembly is located under the cross-bar. The gear is equipped with a handle for manually operating the device and the ratchet (Fig. 1C). The three strings connected to pulleys are fixed to the rotational steel bar.

\section{Stretching protocol}

The patient places the affected hand on the resting hand splint and fixes digits to the finger and thumb holders. Three areas of the affected upper extremity (the middle forearm, wrist joint and proximal portion of metacarpophalangeal $[\mathrm{MCP}]$ joints of the fingers) were fixed using 3 Velcro straps (Fig. 1D, E). By turning the handle attached to the end of the rotational steal bar, tension is applied to the three strings connected to the finger and thumb holders (Fig. 1F). When fingers are fully stretched, the stretched state is fixed with the locking bar above the gear (Fig. 1C). This stretched state is then maintained for 10 minutes. The stretching exercise was performed twice daily for 4 weeks in the intervention group. We provided patients in the intervention group with a checklist and instructed them to complete the checklist after every exercise session.

\section{Clinical evaluation}

MAS scores were used to evaluate the severity of spasticity in the flexor muscles of the 5 MCP joints $[19,20]: 0$, no increase in muscle tone; 1 , slight increase in muscle tone manifested by a catch and release or by minimal resistance at the end of the ROM when the affected part(s) was moved in flexion or extension; $1+$, slight increase in muscle tone manifested by a catch, followed by minimal resistance throughout the remainder (less than half) of the ROM; 2, more marked increase in muscle tone through most of the ROM, but affected part(s) easily moved; 3 , considerable increase in muscle tone, passive movement difficult; 4, affected part(s) rigid in flexion or extension. Categories $1+$ to 4 were designated 2 to 5 for analysis purposes. Spasticities of flexor muscles of the 5 MCP joints in both groups were assessed three times (4 weeks apart) in each patient. The study design is shown in Fig. 2. Two assessments were conducted on patients in the intervention group before starting the stretching program, that is, at 4 weeks before [pre-1] and immediately before [pre-2] starting the stretching program. A 3rd assessment was conducted 1 day after finishing the 4 -week stretching program (post-1). Clinical evaluations were

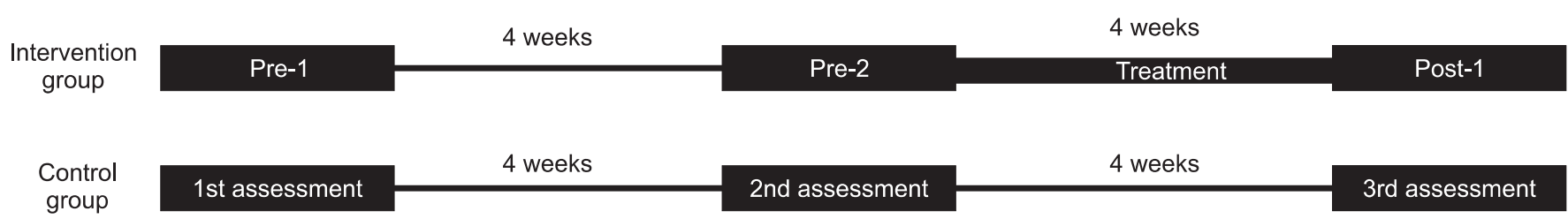

Fig. 2. The study design. 
performed by a physician who was not aware of group identities. The reliability of MAS for joints in the upper extremity has been well demonstrated in several studies [19-22]. Jung et al. [18] also used MAS for assessing changes of spasticity of MCP joint after stretching. The passive range of motion (PROM) was checked at each assessment day in all patients of both groups.

\section{Data analysis}

Data were analyzed using predictive analytics software PASW (IBM Inc., New York, NY, USA). We computed mean MAS (mMAS) scores of flexor muscles for all 5 MCP joints. Demographic data and mMAS scores were compared between the intervention and control groups using the Mann-Whitney U test due to the small number of patients. Changes in mMAS scores with time within groups were evaluated using Wilcoxon's test. Null hypotheses of no difference were rejected if $\mathrm{p}$-values were less than 0.05 .

\section{RESULTS}

No significant intergroup differences were observed for demographic data (Mann-Whitney U test, $\mathrm{p}>0.05$ ) (Table 1). We evaluated intergroup differences of mMAS. Before starting the stretching program, there was no significant difference between mMAS scores at pre-1/pre-2 in the intervention group and those at 1st/2nd in the control group by the Mann-Whitney U test. However, after 4 weeks of the stretching program, mMAS scores at post1 in the intervention group were significantly decreased comparing with the 3rd assessment in the control group (Mann-Whitney U test, $\mathrm{p}<0.05$ ) (Table 2).

Serial changes in mMAS scores were evaluated in the two groups. In the intervention group, no significant difference was observed between pre-1 $(2.71 \pm 0.25)$ and pre2 (2.60 \pm 0.32$)$ (Wilcoxon's test, $\mathrm{p}>0.05)$, but mMAS score at post- $1(1.58 \pm 0.36)$ was significantly lower than at pre$2(2.60 \pm 0.32)$ (Wilcoxon's test, $\mathrm{p}<0.05)$. Within the control group, no significant mMAS score changes were observed between 1st $(2.62 \pm 0.52)$, 2nd $(2.64 \pm 0.50)$, and 3rd $(2.57 \pm 0.50)$ assessments (Wilcoxon's test, $\mathrm{p}>0.05$ ) (Table 2, Fig. 3). All patients in both groups showed no limitation in the PROM of MCP joint in each assessment.

\section{DISCUSSION}

In the present study, we evaluated the effect of this modified version of our hand-stretching device and the

Table 1. Patient demographic data

\begin{tabular}{lcccc}
\hline & Intervention group & Control group & Total & p-value \\
\hline Sex (male:female) & $5: 3$ & $5: 2$ & $10: 5$ & 0.955 \\
Age (yr) & $47.7(8.0)$ & $55.1(14.0)$ & $51.2(11.4)$ & 0.152 \\
Affected side (left:right) & $5: 3$ & $2: 5$ & $7: 8$ & 0.281 \\
Duration (day) & $1,562.3(1,553.4)$ & $1,055.4(1,283.3)$ & $1,325.7(1,407.4)$ & 0.281 \\
mMAS at baseline & $2.71(0.25)$ & $2.62(0.52)$ & $2.67(0.39)$ & 0.613 \\
\hline
\end{tabular}

Values are presented as means (standard deviations).

Duration, duration from onset to first clinical assessment; mMAS, mean Modified Ashworth Scale score; baseline, first clinical assessment ( 4 weeks before starting treatment in the intervention group or 1st assessment in control group).

Table 2. Mean Modified Ashworth Scale scores at different times in the two study groups

\begin{tabular}{lccccc}
\hline \multicolumn{1}{c}{ Group } & $\begin{array}{c}\text { Pre-1/1st } \\
\text { assessment }\end{array}$ & p-value $^{\text {a) }}$ & $\begin{array}{c}\text { Pre-2/2nd } \\
\text { assessment }\end{array}$ & p-value $^{\text {b) }}$ & $\begin{array}{c}\text { Post-1/3rd } \\
\text { assessment }\end{array}$ \\
\hline Intervention & $2.71(0.25)$ & 0.188 & $2.60(0.32)$ & $0.008^{*}$ & $1.58(0.36)$ \\
Control & $2.62(0.52)$ & 0.750 & $2.64(0.50)$ & 0.406 & $2.57(0.50)$ \\
p-value $^{c)}$ & 0.613 & - & 0.955 & - & $0.001^{*}$ \\
\hline
\end{tabular}

Values are presented as means (standard deviations).

${ }^{a)} \mathrm{p}$-value of difference of MAS between pre-1/1st assessment and pre-2/2nd assessment in each group, ${ }^{\text {b) }}$ p-value of difference of MAS between pre-2/2nd assessment and post-1/3rd assessment in each group, ${ }^{\mathrm{c}} \mathrm{p}$-value of difference of MAS between intervention group and control group at each assessment.

${ }^{*} \mathrm{p}<0.05$. 


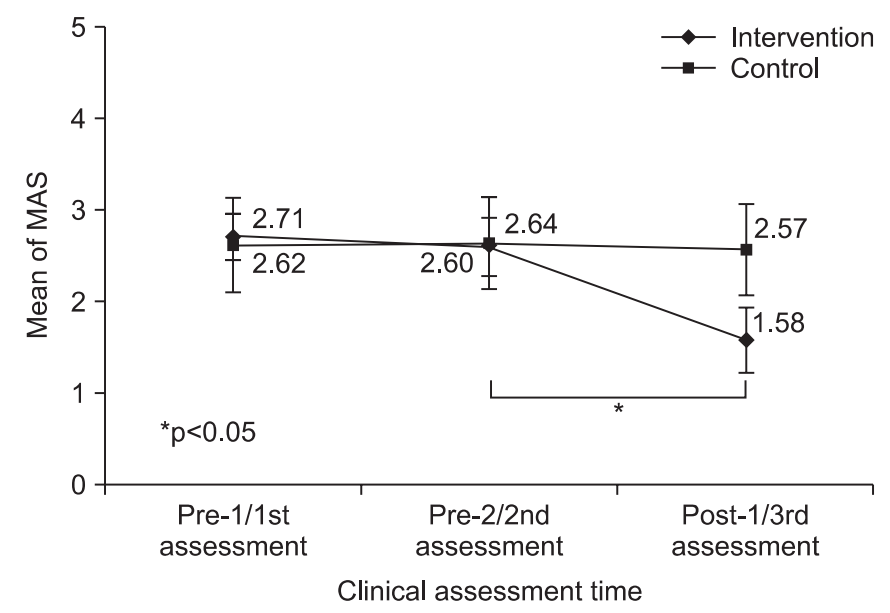

Fig. 3. Sequential changes in mean Modified Ashworth Scale (MAS) scores in the two study groups.

management protocol for the treatment of hand spasticity in chronic hemiparetic stroke patients. After 4 weeks of the stretching program in the intervention group, mMAS scores were significantly reduced, whereas no change was observed in the control group. This result means that the modified device and protocol used in this study effectively reduced hand spasticity in chronic hemiparetic stroke patients. Furthermore, all patients complied with and completed the treatment and no side effects were reported.

Stretching exercise is known to effectively reduce spasticity [13,23-25] by increasing tissue extensibility [26]. Only four studies have been conducted on specifically designed stretching devices. In 2002, Zhang et al. [17] developed an intelligent stretching device that could safely stretch an ankle to a specific torque level, and demonstrated that the device was effective at increasing active and passive ROM of the ankle joint and reducing ankle joint stiffness in chronic stroke patients. In 2005, these workers investigated the effectiveness of the same stretching device for the treatment of ankle spasticity in 10 chronic stroke patients, and found that the device had a positive influence on passive ROM of the ankle joint, maximum voluntary contraction, comfortable walking speed, and ankle stiffness [13]. In 2006, Wu et al. [14] developed a device that helped stroke patients conduct repeated, dynamic, passive ankle joint motion exercise in the standing position. They reported that the device was effective at reducing ankle spasticity and improving gait ability, based on a before and after exercise comparison in 12 chronic stroke patients. In 2011, our research team, Jung et al. [18] demonstrated the effectiveness of a handstretching device for the management of hand spasticity in 21 chronic stroke patients. However, their device and stretching protocol had problems, described above, which limited the usefulness of the device in stroke patients. In the present study, we describe a modification of the previously developed device and a simplified protocol, and demonstrate the effectiveness of this combination for the management of hand spasticity in chronic stroke patients. Furthermore, the present study confirms excellent compliance with the treatment protocol and the safety of the described stretching device.

This study is limited in that we recruited only patients with nearly complete hemiplegia. Further studies are warranted to investigate whether our device is effective in patients with incomplete hemiplegia.

In conclusion, we found that the modified stretching device with the simplified stretching protocol effectively relieved hand spasticity in chronic hemiparetic stroke patients. Accordingly, we believe that the described stretching device and protocol could be used to relieve hand spasticity in chronic stroke patients. However, we did not check serial changes in MAS scores during the 4-week treatment period, and we did not investigate the long-term effects of treatment, and thus, further complementary studies are necessary.

\section{CONFLICT OF INTEREST}

No potential conflict of interest relevant to this article was reported.

\section{REFERENCES}

1. Lance JW. What is spasticity? Lancet 1990;335:606.

2. Gallichio JE. Pharmacologic management of spasticity following stroke. Phys Ther 2004;84:973-81.

3. Sahrmann SA, Norton BJ. The relationship of voluntary movement to spasticity in the upper motor neuron syndrome. Ann Neurol 1977;2:460-5.

4. O'Dwyer NJ, Ada L, Neilson PD. Spasticity and muscle contracture following stroke. Brain 1996;119(Pt 5):1737-49.

5. Landau WM. Spasticity: the fable of a neurological demon and the emperor's new therapy. Arch Neurol 
1974;31:217-9.

6. Jang SH, Ahn SH, Park SM, Kim SH, Lee KH, Lee ZI. Alcohol neurolysis of tibial nerve motor branches to the gastrocnemius muscle to treat ankle spasticity in patients with hemiplegic stroke. Arch Phys Med Rehabil 2004;85:506-8.

7. Jang SH, Park SM, Kim SH, Ahn SH, Cho YW, Ahn MO. The effect of selective tibial neurotomy and rehabilitation in a quadriplegic patient with ankle spasticity following traumatic brain injury. Yonsei Med J 2004;45:743-7.

8. Lannin NA, Novak I, Cusick A. A systematic review of upper extremity casting for children and adults with central nervous system motor disorders. Clin Rehabil 2007;21:963-76.

9. Mori F, Koch G, Foti C, Bernardi G, Centonze D. The use of repetitive transcranial magnetic stimulation (rTMS) for the treatment of spasticity. Prog Brain Res 2009;175:429-39.

10. Ng SS, Hui-Chan CW. Transcutaneous electrical nerve stimulation combined with task-related training improves lower limb functions in subjects with chronic stroke. Stroke 2007;38:2953-9.

11. Oki A, Oberg W, Siebert B, Plante D, Walker ML, Gooch JL. Selective dorsal rhizotomy in children with spastic hemiparesis. J Neurosurg Pediatr 2010;6:353-8.

12. Turk R, Burridge JH, Davis R, Cosendai G, Sparrow O, Roberts HC, et al. Therapeutic effectiveness of electric stimulation of the upper-limb poststroke using implanted microstimulators. Arch Phys Med Rehabil 2008;89:1913-22.

13. Selles RW, Li X, Lin F, Chung SG, Roth EJ, Zhang LQ. Feedback-controlled and programmed stretching of the ankle plantarflexors and dorsiflexors in stroke: effects of a 4-week intervention program. Arch Phys Med Rehabil 2005;86:2330-6.

14. Wu CL, Huang MH, Lee CL, Liu CW, Lin LJ, Chen CH. Effect on spasticity after performance of dynamic-repeated-passive ankle joint motion exercise in chronic stroke patients. Kaohsiung J Med Sci 2006;22:610-7.

15. Pin T, Dyke P, Chan M. The effectiveness of passive stretching in children with cerebral palsy. Dev Med Child Neurol 2006;48:855-62.

16. Tsai KH, Yeh CY, Chang HY, Chen JJ. Effects of a single session of prolonged muscle stretch on spastic muscle of stroke patients. Proc Natl Sci Counc Repub China B 2001;25:76-81.

17. Zhang LQ, Chung SG, Bai Z, Xu D, van Rey EM, Rogers MW, et al. Intelligent stretching of ankle joints with contracture/spasticity. IEEE Trans Neural Syst Rehabil Eng 2002;10:149-57.

18. Jung YJ, Hong JH, Kwon HG, Song JC, Kim C, Park S, et al. The effect of a stretching device on hand spasticity in chronic hemiparetic stroke patients. NeuroRehabilitation 2011;29:53-9.

19. Bohannon RW, Smith MB. Interrater reliability of a Modified Ashworth Scale of muscle spasticity. Phys Ther 1987;67:206-7.

20. Kaya T, Karatepe AG, Gunaydin R, Koc A, Altundal Ercan U. Inter-rater reliability of the Modified Ashworth Scale and modified Modified Ashworth Scale in assessing poststroke elbow flexor spasticity. Int J Rehabil Res 2011;34:59-64.

21. Mehrholz J, Major Y, Meissner D, Sandi-Gahun S, Koch R, Pohl M. The influence of contractures and variation in measurement stretching velocity on the reliability of the Modified Ashworth Scale in patients with severe brain injury. Clin Rehabil 2005;19:63-72.

22. Gregson JM, Leathley M, Moore AP, Sharma AK, Smith TL, Watkins CL. Reliability of the tone assessment scale and the Modified Ashworth Scale as clinical tools for assessing poststroke spasticity. Arch Phys Med Rehabil 1999;80:1013-6.

23. Bressel E, McNair PJ. The effect of prolonged static and cyclic stretching on ankle joint stiffness, torque relaxation, and gait in people with stroke. Phys Ther 2002;82:880-7.

24. Nuyens GE, De Weerdt WJ, Spaepen AJ Jr, Kiekens C, Feys HM. Reduction of spastic hypertonia during repeated passive knee movements in stroke patients. Arch Phys Med Rehabil 2002;83:930-5.

25. Harvey LA, Batty J, Crosbie J, Poulter S, Herbert RD. A randomized trial assessing the effects of 4 weeks of daily stretching on ankle mobility in patients with spinal cord injuries. Arch Phys Med Rehabil 2000;81:1340-7.

26. Monaghan K, Horgan F, Blake C, Cornall C, Hickey PPM, Lyons BE, et al. Physical treatment interventions for managing spasticity after stroke. Cochrane Database Syst Rev 2011;7:1-25. 